\title{
Guidelines and Choosing wisely: to do's and not to do's
}

\author{
Hermann Amstad ${ }^{a}$, Jean-Michel Gaspoz ${ }^{\text {, }}$, Lukas Zemp ${ }^{\mathrm{c}}$ \\ ${ }^{a}$ Dr, Secrétaire général de I'ASSM; ${ }^{b}$ Prof. Dr, Président du Directoire de la SSMI; ${ }^{c}$ Secrétaire général de la SSMI
}

Les guidelines ou guides de pratique et les listes "Choosing wisely" qui reposent sur des bases scientifiques solides sont de grande importance dans le quotidien des médecins et contribuent largement à la sécurité des patients en Suisse. L’ASSM projette d'élaborer, en 2015, un concept global de guides de pratique et de listes «Choosing wisely», en collaboration avec l'ASQM et diverses sociétés de disciplines.

Dans leur feuille de route «Un système de santé durable pour la Suisse», publiée fin 2012, les Académies suisses des sciences déclarent, sous l'objectif 5, que «les prestations médicales dans les domaines de la prévention, du diagnostic, de la thérapie et de la réhabilitation ne sont remboursées que si elles satisfont aux critères EAE»; les Académies invitent ainsi les sociétés de discipline à établir, à l'instar du projet "Choosing wisely" de l'American Board of Internal Medicine (ABIM), une liste de dix interventions manifestement inutiles qui ne devraient plus être ni pratiquées ni remboursées.

Où se situent les problèmes? Quels soutiens seraient utiles? Pour qui existe-t-il des guides pratiques et des listes?

\section{L'idée du «Choosing wisely» s'implante en Suisse}

La feuille de route avait prévu la publication d'une telle liste pour 2013. En octobre 2013, la Société Suisse de Pédiatrie (SSP) avait communiqué dans un courrier adressé à l'ASSM qu'un comité avait examiné la liste "Choosing wisely" de l'American Academy of Pediatrics. Mi-mai 2014, la Société Suisse de Médecine Interne générale (SSMI) a lancé, à Genève, sa campagne avant-gardiste "Smarter Medicine» et publié à cette occasion une liste de cinq interventions à éviter, notamment dans le cadre de la pratique en cabinet médical. En 2015, la SSMI devrait publier une nouvelle liste avec cinq interventions concernant le domaine hospitalier. A l'instar du modèle américain, la campagne de la SSMI devrait s'élargir à de nouveaux partenaires issus des milieux des patients et des consommateurs ainsi que des do- maines médicaux, scientifiques, politiques, économiques et administratifs.

\section{Guides de pratique durables: un exemple à suivre}

La feuille de route invite, par ailleurs, les sociétés de disciplines médicales à élaborer des guides de pratique (objectif 5) - une exigence également soutenue par la FMH. Dans un éditorial du Bulletin des médecins suisses [1], Christoph Bosshard, membre du Comité central de la FMH et responsable du ressort «Données, démographie et qualité» (DDQ), souligne que «des guides de pratique appliqués d'une manière adéquate renforcent la prise en charge du patient» et qu'il est important de «réfléchir davantage à la question des guides de pratique».

La FMH respectivement l'ASQM travaillent depuis quelques années déjà sur le thème des guides de pratique. Dès 1999, la FMH avait publié un article intitulé «Guideline pour guidelines». En 2014, elle a confirmé, dans son document de référence, les jalons médicaux et politiques formulés à cette époque. La FMH/l'ASQM tout comme l'ASSM et la SSMI considèrent que la réflexion (auto-)critique au sujet des guides de pratique est capitale pour le bien-être des patients et l'assurance à long terme de la qualité du système de santé suisse.

\section{La robustesse scientifique et le travail en réseau comme pierres angulaires de guides de pratique solides}

L'élaboration de guides de pratique requiert des critères de qualité uniformes et reconnus au niveau international en ce qui concerne les preuves scienti- 
fiques, les conflits d'intérêts, les approches méthodologiques et l'actualité. Les guides de pratique qui ne reposent pas sur des données fiables sont discutables. Les médecins sont confrontés à un défi de taille lors de l'application de guides de pratique valables pour des tableaux cliniques spécifiques à des patients multimorbides; ce thème doit faire l'objet de futures études.

Contrairement à d'autres pays (par ex. l'Allemagne), la Suisse dispose actuellement de peu de guides de pratique ou de lignes directrices. La plupart du temps, les indications précises font défaut ou bien le choix et la définition des guides de pratique sont arbitraires et ne résultent pas de motifs et de choix fondés. L'élaboration de guides de pratique est ambitieuse; elle exige un investissement financier et personnel élevé et leur implémentation soulève souvent des problèmes (voir à ce sujet le document de base de la division DDQ [2]). Dès lors, il est important de motiver et de soutenir activement les sociétés de disciplines suisses dans ce domaine.

\section{Début d'un processus permanent: état des lieux, workshop, publication}

Le 28 avril 2015, un workshop organisé par l'ASSM et l'ASQM sur le thème de "Choosing wisely et guidelines: opportunités et obstacles» se déroulera à Berne; il s'adresse aux représentants de sociétés de disciplines, de réseaux, d'hôpitaux et d'organisations de patients et poursuit les objectifs suivants:

- sensibilisation au potentiel des guides de pratique et des listes "Choosing wisely", dont le but est de garantir des traitements de haute qualité et d'éviter les traitements inutiles;

- sensibilisation aux problèmes soulevés lors de l'élaboration de guides de pratique et de listes "Choosing wisely";

- indication de «best practices", par ex. dans l'optique de la liste Top 5 de la SSMI comme exemple de futurs guides de pratique dans le domaine de la médecine générale interne (MGI).

En prévision du workshop de 2015, l'ASSM a dressé un état des lieux des guides de pratique et des listes,
Campagne "Smarter Medicine» de la

\section{SSMI - un impact à tous les niveaux}

La Société Suisse de Médecine générale Interne (SSMI) a fait œuvre de pionnière en Suisse en lançant, mi-mai 2014, à Genève, la campagne "Smarter Medicine» pour I'optimisation de la qualité et de l'efficacité dans le système de santé suisse. La pierre angulaire de cette campagne est une liste d'examens diagnostiques et de traitements en médecine générale interne (MGI), auxquels il est conseillé de renoncer, parce qu'ils n'offrent aucun bénéfice mesurable pour les patients et présentent plus de risques que d'avantages. La société de discipline reprendra le flambeau en 2015 et poursuivra dans le cadre du $83^{e}$ congrès annuel de la SSMI sur le thème de "Healthy Medicine», du 20 au 22 mai 2015 à Bâle.

La SSMI salue et soutient l'engagement de I'ASSM - son partenaire de la première heure dans la campagne "Smarter Medicine» - dont les objectifs et les visions correspondent à l'engagement des sociétés de disciplines qui œuvrent pour le bien du patient et l'assurance de la qualité dans le système de santé suisse.

www.smartermedicine.ch http://manifestations.ssmi.ch

au moyen d'un questionnaire adressé à différentes sociétés de disciplines médicales, aux principaux réseaux médicaux et aux hôpitaux: quelle est la situation actuelle? Où se situent les problèmes? Quels soutiens seraient utiles? Pour qui existe-t-il des guides de pratique et des listes?

La publication d'une brochure «Guidelines and choosing wisely: to do's and not to do's» est prévue pour l'automne 2015; elle sera distribuée aux sociétés de disciplines, aux réseaux médicaux et aux hôpitaux pour les informer, les sensibiliser et leur transmettre des exemples de «best practices». Il convient également de vérifier l'opportunité de créer un pool financier comme réserve de fonds, en cas de besoin, pour l'élaboration de guides de pratique respectivement d'une liste «Choosing wisely».

\section{Références}

1 Bosshard C. La qualité doit être au service de l'efficacité. Bull Méd Suisses. 2014;95(41):1519.

2 Hostettler S, Kraft E, Bosshard C. RPC - Identifier les critères de qualité. Bull Méd Suisses. 2014;95(3):45-51. 